\title{
Contrasting evidence of the effectiveness of cosmetic surgery from two health related quality of life measures
}

\author{
Anne Klassen, Ray Fitzpatrick, Crispin Jenkinson, Tim Goodacre
}

Cosmetic surgery is a controversial intervention because it is often regarded as a low priority for public provision. ${ }^{1}$ It is increasingly argued that decisions about the public provision of medical and surgical procedures should be made in the light of evidence of effectiveness from patient-based outcome measures. However, very different impressions of effectiveness may be found depending on choice of outcome measure. This short report therefore evaluates two widely used but contrasting approaches to the measurement of health related quality of life to assess the effectiveness of cosmetic surgery.

\section{Method}

New referrals to plastic surgery were recruited if they were at least 16 years of age and consulting a plastic surgeon in the Oxford region for cosmetic surgery operations of the breast, chest, nose, ear or abdomen. ${ }^{2}$ Postal questionnaires were sent to subjects before (that is, baseline) and six months after surgery. Non-respondents were followed up with two reminders sent at two week intervals. The postal questionnaire contained two measures of health related quality of life: the Short Form 36 Health Survey Questionnaire (SF-36), ${ }^{3}$ and the EuroQol EQ-5D. ${ }^{4}$ The SF-36 is a 36 item measure that assesses eight dimensions of health: physical functioning; role limitations because of physical problems; role limitations because of emotional problems; social functioning; mental health; energy; pain; and general health perception. The EQ-5D is a five item measure that can be summed to produce a single score, or "index" value, for health related quality of life. Items of this instrument ask about mobility, self care, usual activities, pain or discomfort and anxiety or depression.

OX1 1NF

R Fitzpatrick

Health Services Research Unit, University of Oxford, Institute of Health Sciences, Headington, Oxford

C Jenkinson

Department of Plastic and Reconstructive Surgery, Radcliffe Infirmary, Oxford T Goodacre

Correspondence to: Professor R Fitzpatrick.

Accepted for publication 15 January 1999

Results

Questionnaires were sent to a total of 656 cosmetic surgery patients consulting for a variety of surgical procedures including breast reduction, rhinoplasty, and abdominoplasty. At six month follow up, 286 patients had undergone surgery and, of these, 198 patients responded to the follow up questionnaire.

To compare the performance of the two measures of health related quality of life, patients were divided into three sub-groups on the basis of EQ-5D scores. Three groups were formed by calculating the difference between their baseline and follow up scores on EQ-5D, resulting in one group whose scores improved between these two observations, a second group whose scores stayed the same, and a third group whose scores worsened after surgery. Then, an analysis was performed of how much change between baseline and follow up was observed on the second measure, the SF-36, in each of the three sub-groups as determined by the first measure, EQ-5D. The magnitude of change in health related quality of life after surgery on SF-36 was computed by effect size statistics. An effect size is calculated as the difference between scores for an instrument at two observations (in this study observations at baseline and six month follow up) divided by the standard deviation at baseline observation. ${ }^{5}$ Using conventional criteria an effect size of 0.2 or less is regarded as small, 0.5 as moderate, and 0.8 or greater as large. Table 1 shows that the sub-group showing improvement on the EQ-5D also improved across all eight domains of the SF-36, with change that ranged from moderate to large in size, and that was statistically significant for all dimensions $(\mathrm{p}<0.001$ on paired $t$ test or Wilcoxon test depending on the distribution of the data). The sub-group with poorer health related quality of life on the EQ-5D at follow up compared with baseline also showed poorer scores (indicated by negative sign) on most dimensions of the SF-36. However, in the sizeable third group, patients classified into the "no change" sub-group on the basis of baseline and follow up scores for EQ-5D, statistically significant changes in terms of improved scores were observed on four SF-36 dimensions - that is, physical functioning, role emotional, mental health, and social functioning.

\section{Discussion}

The EQ-5D has been advocated as an easy to complete tool that can be used to describe and value health related quality of life for economic

Table 1 Changes for the eight dimensions of SF-36 the and postsurgical follow up for patients receiving plastic surgery assessed as improved, staying the same or worse as measured on change scores for EuroQol $E Q-5 D$. Changes for $S F-36$ are expressed in terms of effect size (difference between baseline and follow up score divided by standard deviation of baseline score, with a negative sign indicating deterioration; $n=186$ because of incomplete data)

\begin{tabular}{llll}
\hline & $\begin{array}{l}\text { Improved } \\
(n=80)\end{array}$ & $\begin{array}{l}\text { Same } \\
(n=71)\end{array}$ & $\begin{array}{l}\text { Worse } \\
(n=35)\end{array}$ \\
\hline Physical functioning & 0.57 & 0.31 & -0.02 \\
Role - physical & 0.39 & 0.01 & -0.24 \\
Role - emotional & 0.47 & 0.36 & -0.17 \\
Social function & 0.67 & 0.37 & -0.26 \\
Mental health & 0.75 & 0.32 & 0.02 \\
Energy & 0.78 & 0.17 & -0.32 \\
Pain & 0.79 & 0.06 & -0.72 \\
General health perception & 0.51 & 0.08 & -0.17 \\
\hline
\end{tabular}


evaluations in the form of cost-utility studies of interventions. In this study when the results from the EQ-5D were compared with the SF-36, it became apparent that different interpretations could be gained of health related quality of life changes arising from cosmetic surgery from the two instruments. A substantial proportion of patients was found to experience no change at all according to EQ-5D while experiencing significant improvements on important dimensions of the SF-36. Reliance on evidence of the EQ-5D alone would result in underestimation of the value of cosmetic surgery to many patients and misleading evidence to inform resource allocation for health care. Overall, patients receiving cosmetic surgery experienced substantial benefits across a number of different dimensions of health related quality of life. ${ }^{2}$ This study shows that they experienced substantial improvements in mental health and role limitations attributable to emotional problems in particular. This is consistent with evidence that such patients experience serious self consciousness, embarrassment, psychological distress, and social handicaps as a result of the problems about their appearance with which they present to cosmetic surgery. ${ }^{6}$ In addition, women presenting for breast reduction surgery in particular, as well as experiencing psychological and social concerns, also report pain, discomfort and limitations in physical activities arising from their breast condition and these problems are substantially alleviated as a result of surgery.

1 Klein R, Redmayne S. Patterns of priorities. (Research paper no 7). Birmingham: National Association of Hospitals and Trusts, 1992 .

2 Klassen A, Jenkinson C, Fitzpatrick R, et al. Patients' health related quality of life before and after aesthetic surgery. $B r$ related quality of life before
F Plast Surg 1996;49:433-8

3 Ware JE, Sherbourne CD. The MOS 36-item Short-Form Health Survey (SF-36) I. Conceptual framework and item selection. Med Care 1992;30:473-83.

4 EuroQol Group. EuroQol EQ-5D User Guide. Rotterdam: Centre for Health Policy and Law, Erasmus University, 1996.

5 Kazis L, Anderson J, Meenan R. Effect sizes for interpreting changes in health status. Med Care 1989;27 (supp):S17889.

6 Klassen A, Jenkinson C, Fitzpatrick R, et al. Measuring quality of life in cosmetic surgery patients with a condition-
specific instrument: the Derriford Scale. Br f Plast Surg 1998;51:380-4. 